COLABORACIÓN ESPECIAL

\title{
PLAN DE VIGILANCIA DE BROTES EPIDÉMICOS RECIENTES DE SARAMPIÓN Y RUBEOLA EN CATALUÑA
}

\author{
Mireia Jané, Nuria Torner, $M^{\mathrm{a}}$ José Vidal
}

Subdirección General de Vigilancia y Respuesta a Emergencias de Salud Pública, Agencia de Salud Pública de Cataluña

\section{RESUMEN}

El sarampión y la rubeola son dos enfermedades inmunoprevenibles. En Cataluña, desde 1988, se administra a todos los niños y niñas dos dosis de vacuna antirubeola y antisarampión con altas coberturas vacunales. Desde 1990 se lleva a cabo el Programa de eliminación del sarampión en Cataluña que contempla alcanzar y mantener un nivel elevado de inmunidad en la población mediante coberturas vacunales altas, una vigilancia epidemiológica intensa y una respuesta inmediata ante la aparición de un caso o un brote La tasa de sarampión se situó en 2014 en 1,9 casos/100.000 habitantes, destacando los brotes recientes de 2006, 2011, 2013 y 2014, que afectaron a $381,289,31$ y 124 personas respectivamente. Todos los brotes fueron desencadenados por un caso importado. En el 2011 y 2014 el $6 \%$ y $5,5 \%$ de los afectados fueron profesionales de la salud. En todos los brotes se observó una amplia variedad genotípica. En el caso del Programa de eliminación de la rubeola, desde el 2002 ha habido 68 casos confirmados de rubeola postnatal y 5 casos de rubeola congénita confirmada. En la vigilancia y control del sarampión y la rubeola, además de reforzar las coberturas vacunales, es esencial la declaración urgente, antes de las 24 horas, de la sospecha y la confirmación por parte del laboratorio de los casos declarados. Respecto a las coberturas vacunales, destaca la necesidad de reforzar la vacunación en el grupo de profesionales de la salud al igual que en otros colectivos no vacunados. En este sentido se aconseja vacunar a todas las personas nacidas después de 1966 que no estén correctamente vacunadas con dos dosis de triple vírica. Asimismo es de destacar que el avance en el estudio de los genotipos aislados permite identificar diversas importaciones procedentes de otros países europeos con brotes activos, aspecto que facilita la vigilancia de estas enfermedades.

Palabras clave: Brotes epidémicos. Sistema de información. Sarampión. Rubéola. Vacunas.

Correspondencia

Mireia Jané Checa

Subdirección General de Vigilancia y Respuesta a Emergencias de Salud Pública

Agencia de Salud Pública de Cataluña

Roc Boronat, 81-95

08005 Barcelona

mireia.jane@gencat.cat

\section{ABSTRACT Surveillance Plan on Recent Outbreak of Measles and Rubella in Catalonia, Spain}

Measles and rubella are two immuno-preventive illnesses. In Catalonia, since 1988 all children are given two doses of measles and rubella vaccine with high levels of vaccination coverage. The measles elimination programme has been carried out since 1990 in Catalonia. This programme includes achieving and keeping high immunization levels among population with high vaccination coverage, intense epidemiological surveillance and an immediate response to the appearance of a case or outbreak. In 2014 , the measles incidence rate was 1.9 cases/ 100,000 inhabitants. There were 4 recent outbreaks in 2006, 2011, 2013 and 2014 that affected 381, 289, 31 and 124 people respectively. All outbreaks were triggered by an imported case. In 2011 and 2014 measles outbreaks, $6 \%$ and $5.5 \%$ of affected people were health care workers. All outbreaks presented a great variety of measles genotypes. Concerning rubella elimination programme, since 2002, 68 cases of postnatal rubella and 5 cases of congenital rubella were confirmed. Regarding measles and rubella surveillance and control, in addition to strengthen vaccination coverage, it is essential immediate notification, within the first 24 hours since suspicion and laboratory confirmation. In addition there is a need to enforce vaccination among health care workers as well as in other susceptible and unvaccinated people. It is recommended to vaccinate all people who were born after 1966 and who have not been vaccinated with two doses of trivalent measles-mumps-rubella vaccine. Furthermore, we have to emphasize that the progress concerning genotypes study allows identifying various imported cases from other European countries with active outbreaks, aspect that makes easier the surveillance of these illnesses.

Keywords: Outbreaks. Information system. Measles. Rubella. Vaccines. Vaccination

DOI: 


\section{INTRODUCCIÓN}

Cataluña es una comunidad autónoma con una gran proporción de población inmigrante, el $14,5 \%$ sobre una población postcensal que se sitúa en 7.426.222 habitantes ${ }^{1}$. Este porcentaje llega a situarse en el 17\% en áreas con mayor densidad de población, como Barcelona y su área metropolitana. Cabe destacar la alta globalización que presenta, con gran movimiento de personas, donde todo está interconectado y los cambios se producen muy rápidamente ${ }^{2}$. Así en 2014, pasaron por el aeropuerto del Prat 37,5 millones de personas ${ }^{3}$. Este voluminoso número de viajeros, junto a la masiva expansión del comercio internacional, el impacto ambiental de la actividad humana diaria y los cambios climáticos, entre otros aspectos, influyen en la rápida propagación de enfermedades y otras amenazas de salud pública ${ }^{4}$.

El sarampión y la rubeola son dos enfermedades immunoprevenibles. La vacuna de la rubeola se introdujo en el calendario de vacunación sistemático de la Generalitat de Cataluña en 1978 para todas las niñas de 11 años de edad $^{5,6}$. En 1981 se introdujo la vacuna triple vírica (sarampión, rubeola y parotiditis) para los niños y niñas al cumplir el año de vida, aunque en 1987 se retrasó hasta los 15 meses de edad. En 1988 se sustituyó la vacuna contra la rubeola que se administraba a los 11 años de edad por la vacuna triple vírica. En 1999 se avanzó la edad de administración de la segunda dosis de vacuna triple vírica y se pasó de los 11 años a los 4 y, finalmente, en 2007 se avanzó la primera dosis de los 15 meses a los 12 meses de edad. Por tanto, en Cataluña desde el año 1988 se administran a todos los niños y niñas dos dosis de vacuna antirubeola y antisarampión siendo las coberturas vacunales altas.

En definitiva, la primera dosis de rubeola se introdujo en las niñas en 1978 y la segunda dosis en 1988. En los niños, la primera dosis se introdujo en 1981 y la segunda en 1988. Con la administración de estas dos dosis de vacunación lo que se pretende no es incrementar el título de anticuerpos de las personas vacunadas sino su cobertura.
La cobertura vacunal del sarampión en 2014 se situó en el 90,91\% para la primera dosis y en el $88,51 \%$ para la segunda. Cabe mencionar que hay una infraestimación en la cobertura, especialmente en las áreas donde hay una proporción significativa de pediatras privados que administran vacunas y donde estas acciones no quedan registradas en los servicios de atención primaria del sistema nacional de salud.

\section{PROGRAMA DE ELIMINACIÓN DE SARAMPIÓN Y RUBEOLA EN CATALUÑA}

Es un hecho universal demostrado y aceptado que las vacunas son una actuación de prevención primaria de gran valor en salud pública, especialmente para aquellas enfermedades que disponen de una vacuna de eficacia elevada y cuyo reservorio es exclusivamente humano, lo que permite una inmunización de la población que contribuye a romper la cadena epidemiológica de la enfermedad e interrumpir la transmisión a la comunidad ${ }^{7}$.

Debido a que el sarampión reúne las condiciones óptimas para ser eliminado de una población o país, el Departament de Salut puso en marcha en 1990, el Programa de eliminación del sarampión en Cataluña. Para poder alcanzar el objetivo se adoptaron estrategias entre las que se encuentra adelantar la administración de la segunda dosis de vacuna a los 4 años de edad, a partir del año 1999, en vez de administrarla a los 11 años, como se hacia anteriormente. En este sentido todo programa de eliminación de sarampión y rubeola de una determinada comunidad ha de tener en cuenta las estrategias de un plan de eliminación ${ }^{8,9}$ :

1. Alcanzar y mantener un nivel elevado de inmunidad de la población infantil mediante una cobertura vacunal elevada con dos dosis de vacuna de sarampión y rubeola.

2. La vigilancia epidemiológica como eje principal del programa de eliminación del sarampión. Cuanto más bajo sea el número de casos, más intensa ha de ser la vigilancia epidemiológica ${ }^{10,11}$. 
3. Una respuesta inmediata ante la aparición de un caso o brote.

La cobertura vacunal del sarampión es alta, alrededor del $90 \%$ en las dos dosis ${ }^{12}$.

Ante un caso clínicamente compatible con sarampión se ha de declarar de forma urgente (antes de 24 horas) a la unidad de vigilancia epidemiológica correspondiente al ámbito territorial donde ejerce el médico/a declarante, con la finalidad de iniciar las actuaciones de control de manera inmediata y la coordinación de las pruebas de laboratorio para confirmar el caso ${ }^{13}$. Las actuaciones son coordinadas por las diferentes unidades de vigilancia epidemiológica del territorio y se reportan a la Subdirección General de Vigilancia y Respuesta a Emergencias de Salud Pública. El seguimiento de los casos y de los contactos y la recogida de las muestras clínicas se harán entre los días 0 y 28 posteriores al inicio del exantema.

En todos los casos se hace una encuesta epidemiológica detallada para conocer la historia inmunológica y el número de dosis administradas de vacuna triple vírica, con el fin de identificar la fuente de infección y conocer dónde y cuándo tuvo lugar la transmisión, lo que facilita la investigación de contactos. Esta encuesta incluye la confirmación clínica, epidemiológica y de laboratorio del caso así como la identificación de la población con riesgo de contraer la enfermedad. Conviene identificar a las personas susceptibles que iniciaron el contacto durante el período de transmisibilidad de la enfermedad y en caso que no acrediten inmunidad frente a la enfermedad deberán recibir la vacuna triple vírica ${ }^{14}$. Igualmente, la rubeola postnatal se rige por los mismos principios de eliminación que para el sarampión y, por lo tanto, las actuaciones de vigilancia y control se llevan a cabo en paralelo desde que se implementó el protocolo para la eliminación de la rubeola postnatal en Cataluña.
En resumen, la vigilancia se nutre de cuatro fuentes de información: el sistema de declaración de enfermedades obligatorias, el cribado de sarampión y rubeola conjunto ante cualquier sospecha, el Sistema de Notificación Microbiológica de Cataluña (SNMC) y el sistema de información basado en el conjunto mínimo básico de datos de las altas hospitalarias (CMBD).

El sistema de notificación de enfermedades de declaración obligatoria de Cataluña, por decreto legislativo ${ }^{13}$, mediante el establecimiento de procedimientos de notificación y de las responsabilidades de control sanitario de las distintas unidades de vigilancia epidemiológica y el ámbito asistencial, permite notificar las enfermedades de declaración obligatoria y los brotes epidemiológicos, entre ellos los casos sospechosos de sarampión y rubeola congénita y posnatal.

En cuanto al cribado, desde el año 1998 a cualquier sujeto con erupción, fiebre y síntomas respiratorios se le investigarán la presencia de virus mediante técnica de reacción en cadena de la polimerasa a tiempo real (RT-PCR), de anticuerpos IgM específicos y, desde 2007, también genotipado de los virus a partir de las secuencias amplificadas por PCR para sarampión y rubeola. En los casos negativos a ambos virus se realizan pruebas de diagnóstico diferencial con otros virus exantemáticos (virus del herpes humano $6 \mathrm{y}$ parvovirus B19). El sistema de notificación microbiológica de Cataluña permite obtener información sobre las detecciones positivas de anticuerpos IgM específicos y de detección de antígenos virales por PCR. Este sistema cubre un gran número de centros que representan más del $80 \%$ de las camas hospitalarias. Asimismo, en el marco del sistema de registro del conjunto mínimo básico de datos se realiza una revisión del registro de las altas hospitalarias por semestre para rubeola, síndrome de rubeola congénita y sarampión de acuerdo con la clasificación internacional de enfermedades de la $9^{a}$ revisión. 


\section{Figura 1}

Tasa de incidencia anual de casos de sarampión por 100.000 habitantes. Cataluña, 1998-2014

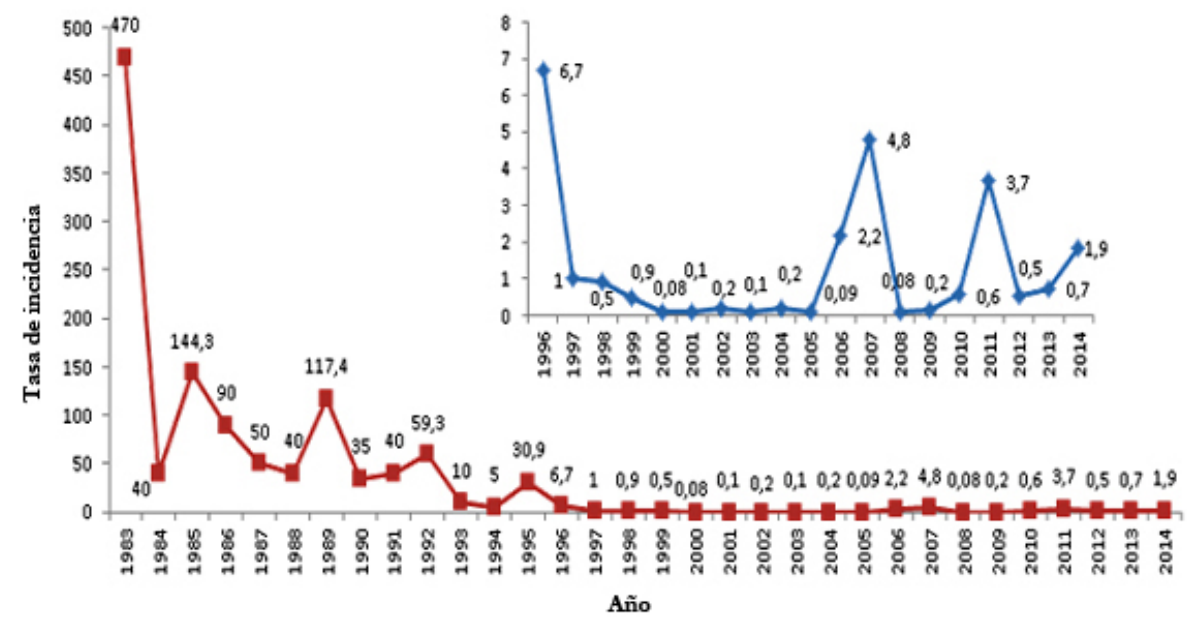

Fuente: Subdirección General de Vigilancia y Respuesta a Emergencias de Salud Pública. Agencia de Salud Pública de Cataluña.

RESULTADOS DEL PROGRAMA DE ELIMINACIÓN DE SARAMPIÓN. CATALUÑA, 2010-2014

Como ya se ha comentado, en Cataluña, la vacuna triple vírica se introdujo en año 1981 en el calendario de vacunas sistemáticas, llegando en el 1982 a una cobertura vacunal del $90 \%{ }^{15}$. Antes de 1980 estaba disponible la vacuna antisarampión monovalente. Como consecuencia de la introducción de esta nueva vacuna se produjo una importante disminución de casos de sarampión que, a pesar de un repunte que se produjo en el año 1989, continuó disminuyendo desde una tasa de 470 casos de sarampión/100.000 habitantes en Cataluña en 1983 hasta una tasa de 1,9 casos/100.000 habitantes en el año 2014. Tal como se observa en la figura 1 destacan en los últimos años 4 brotes relevantes ocurridos en 2006-2007, 2010-2011, 2013 y 2014. El primer brote fue causado por la importación de un virus de genotipo D4 y tuvo una duración en el tiempo de 9 meses, afectando a
381 personas (figura 2). El 50\% de los casos fue en menores de 15 meses y que no estaban todavía vacunados ${ }^{16}$. En consecuencia, en 2007, se avanzó la edad de administración de la primera dosis de vacuna triple vírica a los 12 meses de vida en el calendario de vacunaciones sistemáticas de Cataluña.

Posteriormente, se produjeron dos brotes importantes, uno en el período 2010-2011 y otro en año 2014, que afectaron a 289 y 124 personas respectivamente. El estudio de genotipos aislados permitió identificar en el primer brote múltiples importaciones procedentes de otros países europeos (genotipo D4, D9 y G3) y en el segundo importaciones de otras comunidades autónomas con brotes activos (genotipo B3) ${ }^{17}$. El brote del 2010-2011 afectó a adultos jóvenes no vacunados (figura 3 ).

Entre los brotes de sarampión del 2006 y del 2011 hubo destacadas diferencias ${ }^{18}$. Respecto al brote del 2006, como ya se ha comentado, el $50 \%$ de los casos se dio en lactantes meno- 


\section{Figura 2}

Descripción del brote comunitario de sarampión. Cataluña, 2006-2007

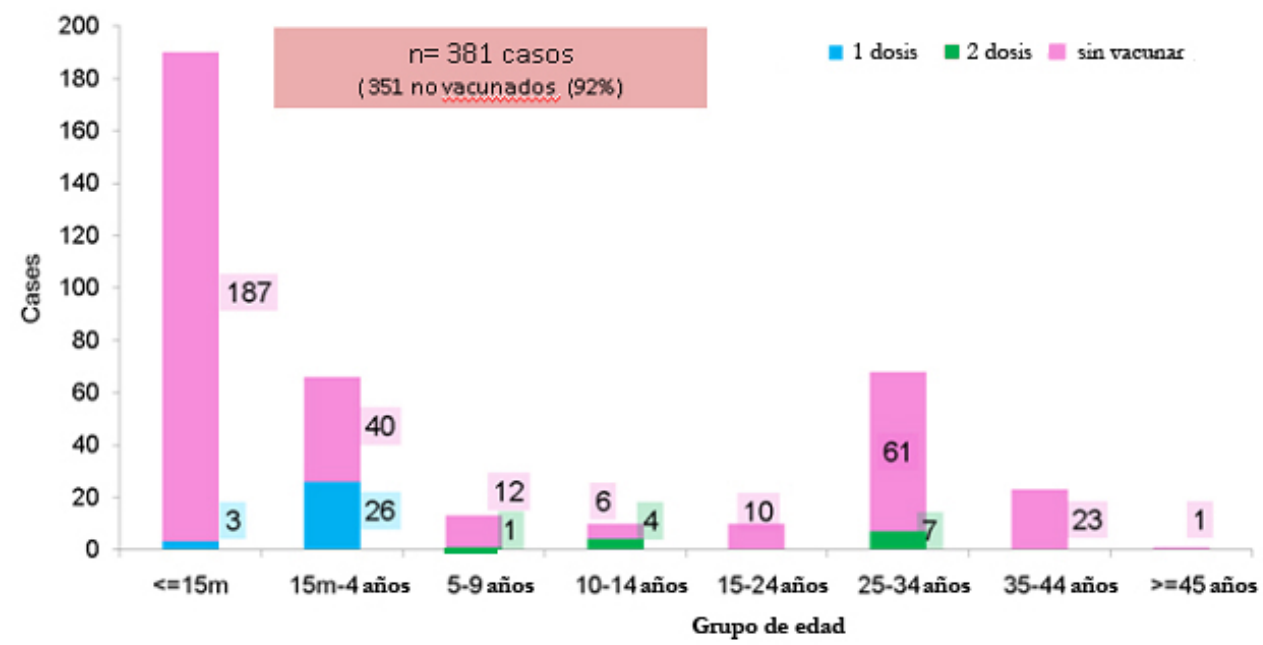

Fuente: Subdirección General de Vigilancia y Respuesta a Emergencias de Salud Pública. Agencia de Salud Pública de Cataluña.

Figura 3

Descripción del brote comunitario de sarampión. Cataluña, 2011

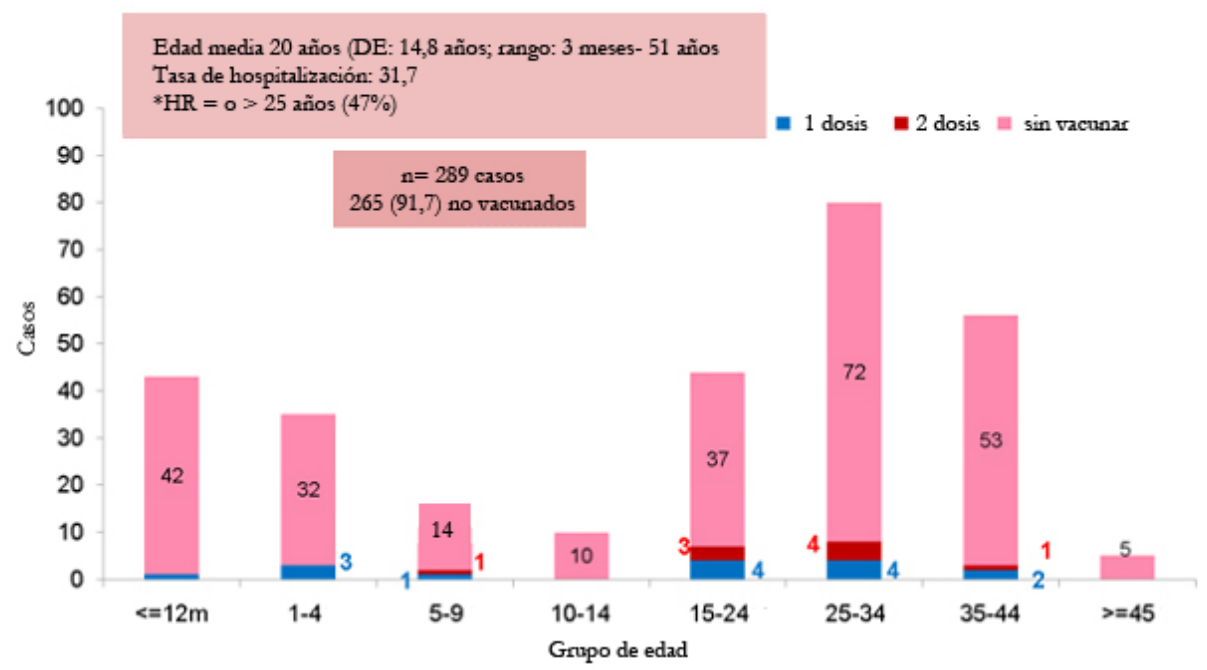

Fuente: Subdirección General de Vigilancia y Respuesta a Emergencias de Salud Pública. Agencia de Salud Pública de Cataluña. 


\section{Figura 4}

Descripción del brote comunitario de sarampión y otras asociaciones de casos.

Cataluña, 2013



Fuente: Subdirección General de Vigilancia y Respuesta a Emergencias de Salud Pública. Agencia de Salud Pública de Cataluña.

res de la edad de vacunación. En cambio en el brote de 2011 solo el 14,8\% afectó a menores de 12 meses de vida. Hay que destacar que el brote del 2006 fue causado por la importación de un virus de genotipo D4 mientras que en el brote del 2011 se identificaron múltiples importaciones genotípicas procedentes de otros países europeos (genotipo D4, D9 y G3). El brote del 2006 tuvo una tasa de hospitalización del 15,7\% mientras que en el de 2011 la tasa fue más alta situándose en un $31,7 \%$. El brote del 2006 afectó a un 3\% de los trabajadores de la salud mientras que en el de 2011 este porcentaje se elevó al 6\%. En ambos la duración se prolongó durante 11 meses y fueron desencadenados por casos importados de otros países y de otras comunidades autónomas. El porcentaje de sujetos no vacunados fue similar en ambos brotes, 92\% en 2006 y $91,7 \%$ en 2011.

El año 2013 se produjeron 54 casos de los cuales 31 estaban asociados a un brote, en el cual el caso índice fue desencadenado por un caso importado de Alemania ${ }^{12}$ y vinculado a un evento musical. Cabe destacar que cada vez hay que tener más en cuenta a la población inmigrante susceptible y los grupos con creencias vinculadas a no vacunar. Los grupos de edad con mayor porcentaje de afectación fueron el de 25 a 34 años y el de 35 a 44 años. El 97\% de los casos no estaba correctamente vacunado y el $5,4 \%$ de las personas afectadas eran profesionales de la salud. La tasa de hospitalización se situó en el $40 \%$, con un caso de encefalitis. Tal como se observa en la figura 4 , el $90 \%$ de las muestras presentaron genotipo D8 MVs/ FrankfurtMain.DEU/17.11/variante. La duración del brote se prolongó durante dos meses.

En 2014 se produjo otro brote de sarampión con 124 casos en el que el caso índice fue desencadenado por un caso importado de Filipinas. El $92,7 \%$ de los sujetos no estaban vacunados, aunque el $10 \%$ era menor de 12 meses de edad por lo que no le correspondía la vacuna. La tasa de hospitalización se situó en el 28,4\%. El 23,4\% de los afectados fueron trabajadores sanitarios. 


\section{Figura 5}

\section{Descripción del brote comunitario de sarampión. Cataluña, 2014}



Fuente: Subdirección General de Vigilancia y Respuesta a Emergencias en Salud Pública. Agencia de Salud Pública de Cataluña.

El $80 \%$ de las muestras tuvieron genotipo B3 MVs/WesternAustralia.AUS/2.14/variante. La duración del brote se prolongó durante cinco meses. Destaca la recomendación dirigida a todos los trabajadores de la salud susceptibles que deberían estar inmunizados contra el sarampión (figura 5).

\section{RESULTADOS DEL PROGRAMA DE ELIMINACIÓN DE LA RUBEOLA. CATALUÑA, 2002-2014}

Ya se ha comentado en apartados anteriores que en 1978 se introdujo en el calendario vacunal de Cataluña la administración de la vacuna antirubeola para las niñas de 11 años. Dos años después, se introdujo la triple vírica a los niños y niñas al cumplir el año de vida, y en 1988 se sustituyó la vacuna antirubeola de los 11 años por la triple vírica. Al año siguiente se adelantó la segunda dosis de triple vírica a los 4 años de edad. Finalmente en 2007 se adelantó la dosis de vacuna de los 15 meses al año de vida. En definitiva, en Cataluña desde el año 1988 se administra a todos los niños y niñas 2 dosis de vacuna antirubeola ${ }^{19}$.

En la figura 6 se muestra la distribución de los casos de rubeola desde 1983 a 2014. Se observa un pico con una tasa de incidencia de 394,5 casos de rubeola /100.000habitantes en el año 1985. Fue a partir de 1988, coincidiendo con la administración de la vacuna triple vírica a niños y niñas de 11 años, cuando empezó a disminuir el número de casos notablemente. Se observa una disminución progresiva en la incidencia de esta enfermedad, pasando de 0,5/100.000 habitantes en 1997 a 0,01/100.000 habitantes en 2014.

La figura 7 muestra la distribución de los casos de rubeola postnatal y congénita sospechosos y confirmados durante el periodo 20022014. 
Figura 6

Tasa de incidencia anual de casos de rubeola por 100.000 habitantes. Cataluña, 1983-2014

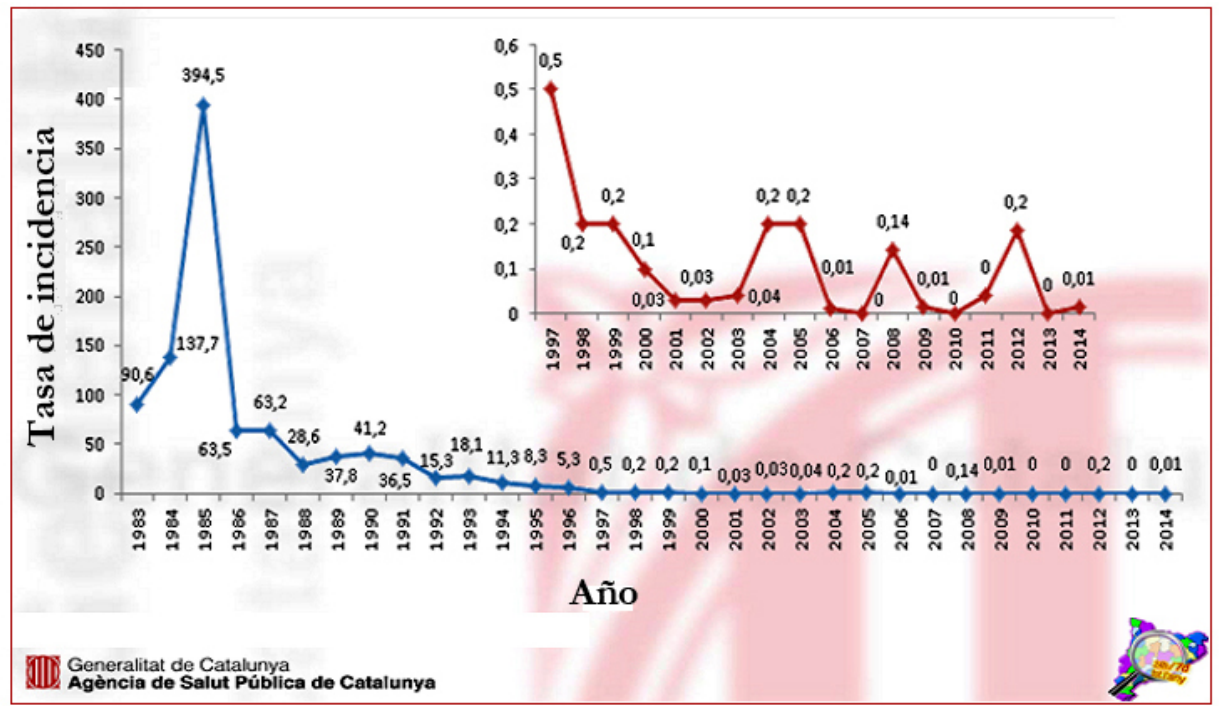

Fuente: Subdirección General de Vigilancia y Respuesta a Emergencias de Salud Pública. Agencia de Salud Pública de Cataluña.

Figura 7

Morbilidad de la rubeola postnatal y rubeola congénita. Cataluña, 2002-2014



2004, 2005*, 2008, $2012,2014 \$$ casos de SRC confirmados

*primer caso autóctono SRC desde 1990

Infección congénita IRC (no presenta SRC)

Fuente: Subdirección General de Vigilancia y Respuesta a Emergencias de Salud Pública. Agencia de Salud Pública de Cataluña. 
Cabe destacar que en los últimos años los casos de rubeola que han aparecido están relacionados con incrementos observados en diversos países europeos, como Ucrania, Polonia y Rumania, aspecto que constata la posibilidad de importación de casos procedentes de zonas donde se encuentra una situación endémica o con coberturas de vacunación bajas.

\section{CONCLUSIONES}

En el contexto de la eliminación del sarampión y la rubeola en Cataluña, además de mantener unas altas coberturas vacunales entre la población, es fundamental la declaración urgente de cualquier sospecha de caso y su confirmación en el laboratorio, ya que solamente así se puede tener precisión de la incidencia real de ambas enfermedades ${ }^{14,20}$. Esta declaración urgente debe hacerse antes de que transcurran 24 horas desde la sospecha. El caso debe comunicarse a la unidad de vigilancia epidemiológica correspondiente.

Las coberturas vacunales de la triple vírica son elevadas en Cataluña, pero en los últimos años se ha detectado un incremento en la frecuencia de brotes de sarampión y se ha observado que todos los brotes han estado desencadenados por casos importados de personas procedentes de países donde las tasas de vacunación son más bajas o donde estas enfermedades todavía están consideradas endémicas. En estos brotes destaca el incremento de casos en profesionales de la salud, lo que demuestra la necesidad de reforzar su vacunación, al igual que incidir en otros sujetos susceptibles no vacunados.

De acuerdo con la línea estratégica de eliminar el sarampión y la rubeola en la región europea, se aconseja vacunar a todas las personas nacidas después de 1966 que no tengan la certeza demostrada de haber pasado la enfermedad o haber estado correctamente vacunadas con dos dosis. Esta recomendación está dirigida especialmente a todas las personas profesionales de la salud y a las que viajen fuera de España.
El avance en el estudio de los genotipos aislados para el sarampión ha permitido identificar múltiples importaciones provenientes de otros países europeos y otras comunidades autonómicas con brotes activos mediante el análisis filogenético de las cepas.

\section{BIBLIOGRAFÍA}

1. Estimacions postcensals de població (EP). 2014. Idescat. Disponible en: http://www.idescat.cat/novetats/?id=2032

2. Saima Aziz Siddiqui. The challenge of emerging infectious disases; high time for integrated global response. J Park Med Assoc. 2011; 61(12):1165-1167

3. Aena. Informe anual. Estadísticas, tráfico de pasajeros, operaciones y carga en los aeropuertos españoles. Disponible en: http://www.aena.es/csee/ccurl/562/194/estadisticas_anual_2014_provisionales.pdf

4. Castillo-Salgado C. Trends and Directions of Global Public Health Surveillance. Epidemiol Rev 2010; 32:93109

5. Evolució del calendari de vacunacions sistemàtiques de Catalunya (període 1980-2014). Butll Epidemiol Catalunya. 2014; Volum XXXV(2): 15-23.

6. Diari Oficial de la Generalitat de Catalunya. Decret 95/2014, d'1 de juliol, pel qual s'estableix el calendari de vacunacions sistemàtiques. Diari Oficial de la Generalitat de Catalunya Núm. 6656 de 3-7-2014

7. Salleras L, Domínguez A. L'eliminació del xarampió a Catalunya per a l'any 2000. Bases científiques i programa. Quaderns de salut pública. Barcelona: Departament de Salut; 1999.

8. Clemens CJ, Strassburg M, Cutts FT, Milstein J, Torel C. Challenges for the global control of measles in the 1990's. A: Kurstak E (ed.). Measles and Poliomyelitis: Vaccines, Immunization and Control. Viena: Springer-Verlag;1993. p. 13-24.

9. Tipphawong J, Wittes R, Van-Ham O. «Measles vaccine, one versus two doses; why and when». A: KURSTAK E (ed.). Measles and Poliomyelitis Vaccines, Immunization and Control. Viena: Springer-Verlag;1993. p. 75-100.

10. Centers for Disease Control and Prevention (CDC). Measles, mumps, and rubella-vaccine use and strategies for elimination of measles, rubella and congenital rubella syndrome and control of mumps: Recommendations of the Advisory Committee on Immunization Practices (ACIP). MMWR Morb Mortal Wkly Rep 1988;47(RR08):1-57.

11. Stroobant A, Lamotte JM, Van Casteren V, Cornelis R, Walckiers D, Colyn Y. Epidemiological surveillance of measles through a network of sentinel general practitioners in Belgium. Int J Epidemiol 1986;15:386-91. 
12. Agència de Salut Pública de Catalunya. Programa d'eliminació del xarampió a Catalunya. Informes anuals. Xarampió 2013-2014 a Catalunya. Barcelona: Agència de Salut Pública de Catalunya;2015.

13. Diari Oicial de la Generalitat de Catalunya. Decret 67/2010, de 25 de maig, pel qual es regula el sistema de notificació de malalties de declaració obligatòria i brots epidèmics al Departament de Salut. Diari Oicial de la Generalitat de Catalunya núm 5666 de 8-7-2010.

14. Departament de Salut. Protocol d'actuació per a la prevenció i el control del xarampió. Subdirecció General de Vigilància i Resposta a Emergències en salut Pública. Barcelona: Departament de Salut; 2012.

15. Salleras L, Domínguez A, Cuesta A, Domínguez V. Eliminación de enfermedades transmisibles prevenibles por vacunas. A: Salleras L (ed.). Vacunaciones Preventivas. 2a ed. Barcelona: Masson;2003. p. 965-76.

16. Domínguez A, Torner N, Barrabeig I, et al. Large outbreak of measles in a community with high vaccination coverage: implications for the vaccination schedule. Clin Infect Dis. 2008; 47(9):1143-9.

17. López-Perea N, Masa Calles J, Castellanos Ruiz T, Almazán Isla J, Torres de Mier MV, Grupo de Trabajo del Plan Nacional de Eliminación del Sarampión y de la Rubeola. Vigilancia del Sarampión, Rubéola y Síndrome de Rubéola Congénita. España 2011. Bol Epidemiol Sem. 2012; 21(6):55-64.

18. Subdirecció General de Vigilància i Resposta a Emergències en Salut Pública. Informe del brot de xarampió de 2010-2011 a Catalunya. Programa d'eliminació del xarampió a Catalunya. Barcelona: Departament de Salut; 2012.

19. Subdirecció General de Vigilància i Resposta a Emergències en Salut Pública. Programa d'eliminació de la rubèola postnatal i congènita a Catalunya. Informe anual. Rubèola 2012 a Catalunya. Barcelona: Departament de Salut; 2013.

20. Organización Mundial de la Salud. Eliminación de sarampión y rubéola, y prevención de la infección congénita por el virus de la rubéola Plan estratégico 2005-2010 para la Región Europea de la OMS. Ginebra: OMS; 2005. 